\title{
Editorial
}

\section{Should we use more Digital Therapy in Clinical Practice?}

\author{
Oswald D. Kothgassner ${ }^{1} \&$ Anna Felnhofer ${ }^{2}$ \\ ${ }^{1}$ Department of Child and Adolescent Psychiatry, Medical University of Vienna \\ 2 Department of Pediatrics and Adolescent Medicine, Medical University of Vienna
}

DOI 10.24989/dp.v2i1.1980

The short answer to our initial question is: We do not know yet, but we should be positive and open minded towards digital technologies in psychological therapy, because they are a powerful instrument to treat over a larger distance, and employ new possibilities and techniques in the therapeutic process. We should have a closer look on efficacy, safety and acceptance (in patients and clinicians) of digital therapy solutions. Both, patients and clinicians face new challenges ranging from data protection issues to unfamiliar therapeutic settings. Clinicians should know the limits of digital therapy and authorities should invest in digitalization of psychotherapeutic interventions. From our perspective, three digital therapeutic settings will fundamentally influence the psychological therapy in future: (1) Internet-based therapy, (2) smartphone-based therapy and (3) Virtual Reality applications. A substantial part of the published studies in the field failed to report side effects, adverse events, and other safety problems - the latter being a problem in psychological therapies per se. Only few studies report these data for specific applications (e.g. Boettcher et al., 2014). Most studies focus solely on the efficacy of the treatment, therefore we illustrate some current reviews in the present editorial and new developments indicating efficacy and promising results of digital therapies.

\section{Internet-based therapy}

Meta-studies regarding Internet-based therapy show heterogeneous outcomes. Some reviews found that Internet-based interventions are effective in reducing the incidence of depression and decreasing anxiety symptoms in adolescents and adults (e.g. Etzelmueller et al., 2020; Reins et al., 2021). This is contradicted by other reviews, which found only small effects in preventing depression (e.g. Rigabert et al., 2020). However, a recent review showed that telehealth applications and Internet-based therapy are useful tools in treating social anxiety (Andersson et al., 2014), post-traumatic stress disorder (PTSD) (Kuhn \& Owen, 2020), and first RCTs show promising results of Internet-based therapy in individuals with obsessive-compulsive disorder (Schröder et al., 2020).

\section{Smartphone-based therapy}

The use of smartphones devices for mental health purposes is rapidly growing, but especially for unguided apps there is a definite lack of evidence. Current meta-analyses suggest moderate effects in the treatment of depressive symptoms, nicotine addiction and social anxiety symptoms; but smartphone treatments seem to have only limited efficacy in treating general anxiety, PTSD, or suicidality (Goreis et al., 2020; Linardon et al., 2019; Weisel et al., 2019). Results for prodromal and early course psychotic symptoms were highly heterogeneous (Camacho et al., 2019). Moreover, a high drop-out rate is reported for smartphone therapies (e.g. Torous et al., 2020).

\section{Table of Contents}

\section{Editorial}

4 Expert Views on Online Therapy

6 Linking the Technology Acceptance Model to Smartphone Use and Smartphone Use Disorder Tendencies: Results from a Survey Study Cornelia Sindermann, René Riedl, Jon D. Elhai \& Christian Montag

19 Internet-Based Psychological Assessment and Intervention During the COVID-19 Pandemic with an Adolescent Transgender Patient - A Case Report Diana Klinger

23 Expositionstherapie mit Virtual Reality bei Blut-, Verletzungs- und Spritzenphobie. Eine Fallstudie Johannes S. Lanzinger, Julia Neukam \& Christian Dingemann 


\section{Virtual Reality therapy}

Virtual Reality has been proven to be an efficient tool for exposure therapy in anxiety disorders with comparable effects to invivo exposure (Carl et al., 2019). Moreover, Virtual Reality exposure therapy seems to be a promising therapeutic approach for the treatment of PTSD (Kothgassner et al., 2019). However, only few studies are carried out including children and adolescents (Kothgassner et al., in press). Nevertheless, Virtual Reality is also used in the therapy of people with mild cognitive impairment and dementia, but has only limited efficacy (Kim, Pang \& Kim, 2019). An innovative approach was used in the treatment of psychosis: an RCT by Pot-Kolder et al. (2018) showed that virtual cognitive behavioral therapy succeeds to reduce paranoid ideations and anxiety; further data suggest that Virtual Reality is a safe treatment for people suffering from psychosis.

\section{Outlook}

Concluding, there is a growing body of literature on psychological treatments using digital applications, the majority of them seem to have moderate to high efficacy. Many studies compare digital treatments with real-life treatments and demonstrate the advantages of digital therapy. Future RCTs should increasingly focus on researching the safety of treatments and hurdles of using digital technologies in a clinical practice setting. Further, it is important to understand the effect of therapeutic guidance in digital therapies because the specific effect of guidance remains unclear. Some authors argue (Reins et al., 2021) that guided therapies are convenient for severe psychopathologies (e.g. major depression), but may have only a limited effect on the efficacy of subthreshold mental disorders.

Digital therapy took an important role during the pandemic crisis in 2020 and continues to do so. As such it could be a critical augmentation for face-to-face social interactions in rural areas or if social contact is no longer possible (e.g. quarantine measures). A still existing hurdle in many countries is the legalization of Internet-based therapy; in Austria and Germany, for instance, psychological and psychotherapeutic services may not be delivered exclusively online, only counselling activities are allowed using the Internet (see Kothgassner \& Felnhofer, 2018). In sum, the efficacy and safety of these digital interventions have to be investigated, and clinicians have to be trained in the usage of these technologies as well as with regards to the requirements of digital therapy approaches before implementing these applications in clinical practice.

In this issue, we ask experts in the field of internet-based therapy on their opinion and expertise regarding this important topic. The urge for digital treatment methods will last after the pandemic and it is important to start implementation, structural planning and specific guidelines for this period. Therefore, Digital Psychology will foster empirical submissions and reviews about efficacy and side effects of innovative digital therapies, as well as case studies of digital interventions. In the current issue - two case studies are presented for an acute internet-based assessment and intervention during the first week of the COVID-19 crisis (Klinger, 2021) and a VR-intervention for blood and injection phobia (Lanzinger et al., 2021). We wish you a pleasant read.

Oswald D. Kothgassner and Anna Felnhofer

Editors-in-Chief

\section{References}

Andersson, G., Cuijpers, P., Carlbring, P., Riper, H., \& Hedman, E. (2014). Guided Internet-based vs. face-to-face cognitive behavior therapy for psychiatric and somatic disorders: a systematic review and meta-analysis. World Psychiatry, 13(3), 288-295.

Boettcher, J., Rozental, A., Andersson, G., \& Carlbring, P. (2014). Side effects in Internet-based interventions for Social Anxiety Disorder. Internet Interventions, 1(1), 3-11.

Camacho, E., Levin, L., \& Torous, J. (2019). Smartphone apps to support coordinated specialty care for prodromal and early course schizophrenia disorders: systematic review. Journal of medical Internet research, 21(11), e16393.

Carl, E., Stein, A. T., Levihn-Coon, A., Pogue, J. R., Rothbaum, B., Emmelkamp, P., ... \& Powers, M. B. (2019). Virtual reality exposure therapy for anxiety and related disorders: A meta-analysis of randomized controlled trials. Journal of anxiety disorders, 61, 27-36.

Etzelmueller, A., Vis, C., Karyotaki, E., Baumeister, H., Titov, N., Berking, M., ... \& Ebert, D. D. (2020). Effects of internet-based cognitive behavioral therapy in routine care for adults in treatment for depression and anxiety: Systematic review and meta-analysis. Journal of medical Internet research, 22(8), e18100.

Florean, I. S., Dobrean, A., Păsărelu, C. R., Georgescu, R. D., \& Milea, I. (2020). The Efficacy of Internet-Based Parenting Programs for Children and Adolescents with Behavior Problems: A Meta-Analysis of Randomized Clinical Trials. Clinical Child and Family Psychology Review, 23(4), 510-528.

Goreis, A., Felnhofer, A., Kafka, J. X., Probst, T., \& Kothgassner, O. D. (2020). Efficacy of self-management smartphone-based apps for post-traumatic stress disorder symptoms: a systematic review and meta-analysis. Frontiers in neuroscience, 14, 3.

Kim, O., Pang, Y., \& Kim, J. H. (2019). The effectiveness of virtual reality for people with mild cognitive impairment or dementia: a metaanalysis. BMC psychiatry, 19(1), 1-10.

Klinger, D. (2021). Internet-Based Psychological Assessment and Intervention During the COVID-19 Pandemic With an Adolescent Transgender Patient - A Case Report. Digital Psychology 2(1), 19-22.

Kothgassner, O. D., \& Felnhofer, A. (in press). Lack of research on efficacy of virtual reality exposure therapy (VRET) for anxiety disorders in children and adolescents. neuropsychiatrie, 1-8.

Kothgassner, O. D. \& Felnhofer, A. (Hrsg.) (2018). Klinische Cyberpsychologie und Cybertherapie. Wien: UTB facultas.wuv.

Kothgassner, O. D., Goreis, A., Kafka, J. X., Van Eickels, R. L., Plener, P. L., \& Felnhofer, A. (2019). Virtual reality exposure therapy for post- 
traumatic stress disorder (PTSD): a meta-analysis. European journal of psychotraumatology, 10(1), 1654782.

Kuhn, E., \& Owen, J. E. (2020). Advances in PTSD treatment delivery: the role of digital technology in PTSD treatment. Current Treatment Options in Psychiatry, 7(2), 88-102.

Lanzinger, J., Neukam, J. \& Dingeman, C. (2021). Expositionstherapie bei Blut-, Verletzungs- und Spritzenphobie mit Virtual Reality: Ein Fallbericht. Digital Psychology 2(1), 23-27.

Linardon, J., Cuijpers, P., Carlbring, P., Messer, M., \& Fuller-Tyszkiewicz, M. (2019). The efficacy of app-supported smartphone interventions for mental health problems: A meta-analysis of randomized controlled trials. World Psychiatry, 18(3), 325-336.

Pot-Kolder, R. M., Geraets, C. N., Veling, W., van Beilen, M., Staring, A. B., Gijsman, H. J., ... \& van der Gaag, M. (2018). Virtual-realitybased cognitive behavioural therapy versus waiting list control for paranoid ideation and social avoidance in patients with psychotic disorders: a single-blind randomised controlled trial. The Lancet Psychiatry, 5(3), 217-226.

Reins, J. A., Buntrock, C., Zimmermann, J., Grund, S., Harrer, M., Lehr, D., ... \& Ebert, D. D. (2021). Efficacy and moderators of internetbased interventions in adults with subthreshold depression: An individual participant data meta-analysis of randomized controlled trials. Psychotherapy and Psychosomatics, 90(2), 94-106.

Rigabert, A., Motrico, E., Moreno-Peral, P., Resurrección, D. M., Conejo-Cerón, S., Cuijpers, P., ... \& Bellón, J. Á. (2020). Effectiveness of online psychological and psychoeducational interventions to prevent depression: Systematic review and meta-analysis of randomized controlled trials. Clinical Psychology Review, 101931.

Schröder, J., Werkle, N., Cludius, B., Jelinek, L., Moritz, S., \& Westermann, S. (2020). Unguided Internet-based cognitive-behavioral therapy for obsessive-compulsive disorder: A randomized controlled trial. Depression and Anxiety, 37(12), 1208-1220.

Thompson, E. M., Destree, L., Albertella, L., \& Fontenelle, L. F. (2021). Internet-based acceptance and commitment therapy: a transdiagnostic systematic review and meta-analysis for mental health outcomes. Behavior Therapy: 52(2), 492-507.

Torous, J., Lipschitz, J., Ng, M., \& Firth, J. (2020). Dropout rates in clinical trials of smartphone apps for depressive symptoms: a systematic review and meta-analysis. Journal of affective disorders, 263, 413-419.

Weisel, K. K., Fuhrmann, L. M., Berking, M., Baumeister, H., Cuijpers, P., \& Ebert, D. D. (2019). Standalone smartphone apps for mental health - a systematic review and meta-analysis. NPJ digital medicine, 2(1), 1-10.

\section{Conflict of Interest}

OK and AF declare no conflict of interest. 